\title{
CLASSIFICATION OF EARLY TYPE STARS ON THE DOMINION ASTROPHYSICAL OBSERVATORY PHOTOMETRIC SYSTEM
}

\author{
S. C. MORRIS and G. HILL \\ Dominion Astrophysical Observatory, Victoria, B.C., Canada \\ and \\ G. A. H. WALKER and H. I. B. THOMPSON \\ Department of Geophysics, University of British Columbia, Vancouver 8, B.C., Canada
}

\begin{abstract}
A classification method has been developed for early-type stars observed on the Dominion Astrophysical Observatory photometric system. Two reddening-independent parameters, $Q(35)$ and $Q(38)$, are used. $Q(35)$ is a measure of the Balmer discontinuity, while $Q(38)$ is a measure of the strength of the upper members of the Balmer series. A preliminary calibration of $Q(35)$ and $Q(38)$ in terms of spectral types and luminosity classes is given, and applications to several groups of stars are shown.
\end{abstract}

A four-channel photometer was put into use by the Dominion Astrophysical Observatory (DAO) in 1968 and has been used in a variety of observational programs since then. The instrument (described in detail by Walker et al., 1970) has two passbands which reproduce $B$ and $V$ of the $U B V$ system (Johnson et al., 1966) and two ultraviolet bands, one 200 wide placed beyond the Balmer discontinuity, the other 150 wide, which includes the upper members of the Balmer series (H9-H16). We designate our passbands in terms of their mean wavelengths, quoted to the nearest hundred angstroms. Thus, 35 refers to the $\lambda 3400-\lambda 3600$ passband; $c(35-44)$ is the colour derived from $\lambda 3400-\lambda 3600$ and $\lambda 3900-\lambda 4900$ passbands; and similarly for $E(35-44)$, etc.

The instrument was designed in the hope that with one instrument we would be able to derive luminosities and temperatures for early-type stars whose light suffers interstellar reddening. In practice we can observe effectively down to $11^{\mathrm{m}}$ with a 16-in. telescope and un-refrigerated photomultipliers. The multichannel nature of the instrument has important advantages in observational efficiency and in relative immunity from the effects of variable grey extinction due to clouds.

In developing a new photometric system our first task was to obtain colours and magnitudes for a set of standard stars. These have recently been published (Hill et al., 1971). In this paper we present a preliminary calibration of the photometric data in terms of spectral types for early-type stars. Ultimately this calibration will be made in terms of $M_{v}$ and $T_{e}$. In what follows we shall describe how the calibration was derived and show how several groups of stars compare with the basic calibration.

In Figure 1 we show a colour-colour plot [c(35-44) vs $c(44-54)]$ for: (a) relatively unreddened standard stars, (b) 'Petrie-Lee' field stars (Petrie and Lee, 1966) of various degrees of reddening and (c) stars in Cepheus IV, an apparently young cluster of large and variable reddening. The ratio $E(35-44) / E(44-54)$ is derived from the slope of the 


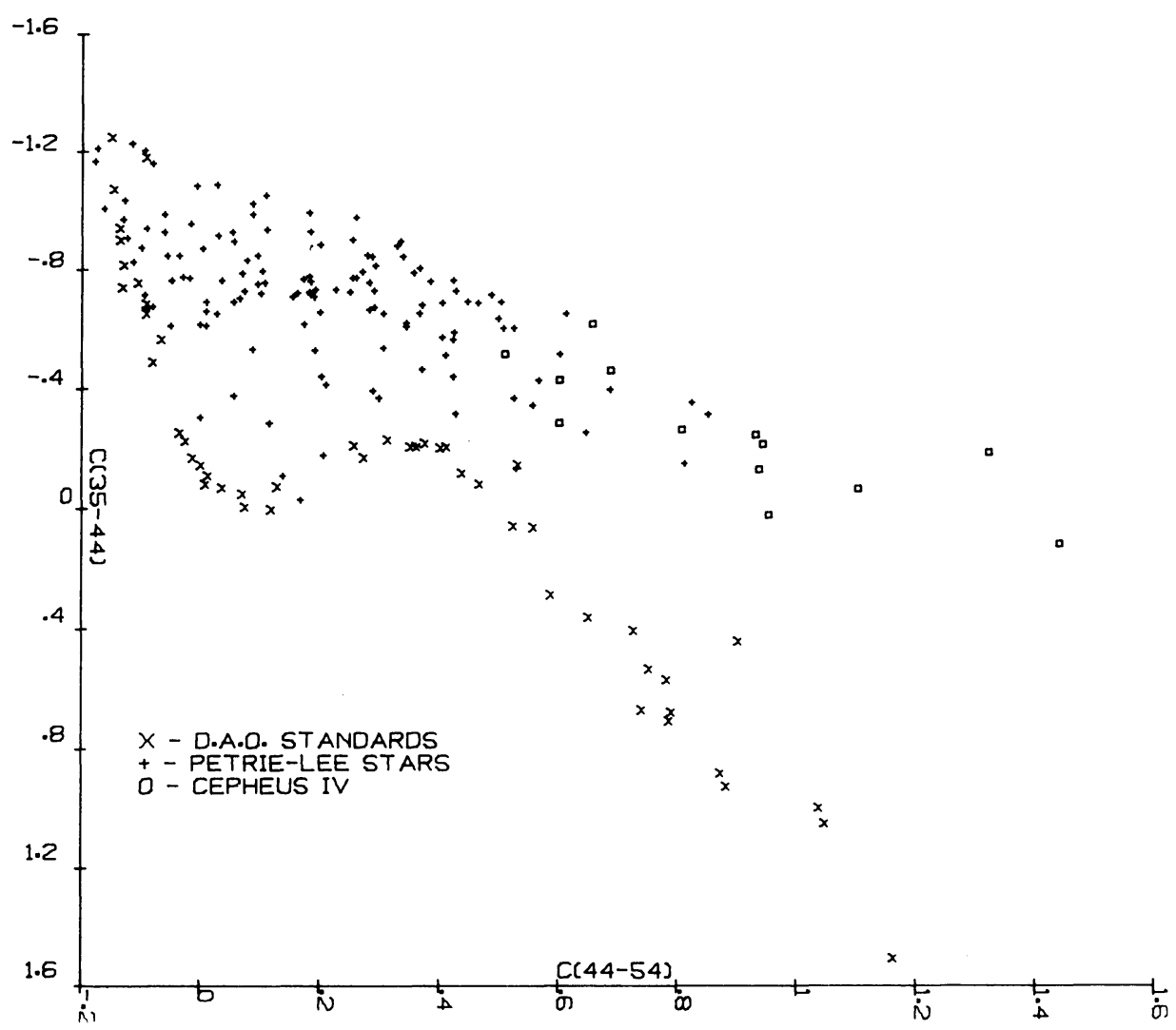

Fig. 1. Colour - colour plot, showing the effects of interstellar reddening.

upper envelope of this plot, and has a value of 0.83 ; the stars in Cepheus IV were not used in this derivation. The ratio $E(38-44) / E(44-54)$ was derived in similar fashion; it is equal to 0.62 .

We define two reddening-free parameters $Q(35)$ and $Q(38)$ by the relations

$$
Q(35)=c(35-44)-\frac{E(35-44)}{E(44-54)} \cdot c(44-54)
$$




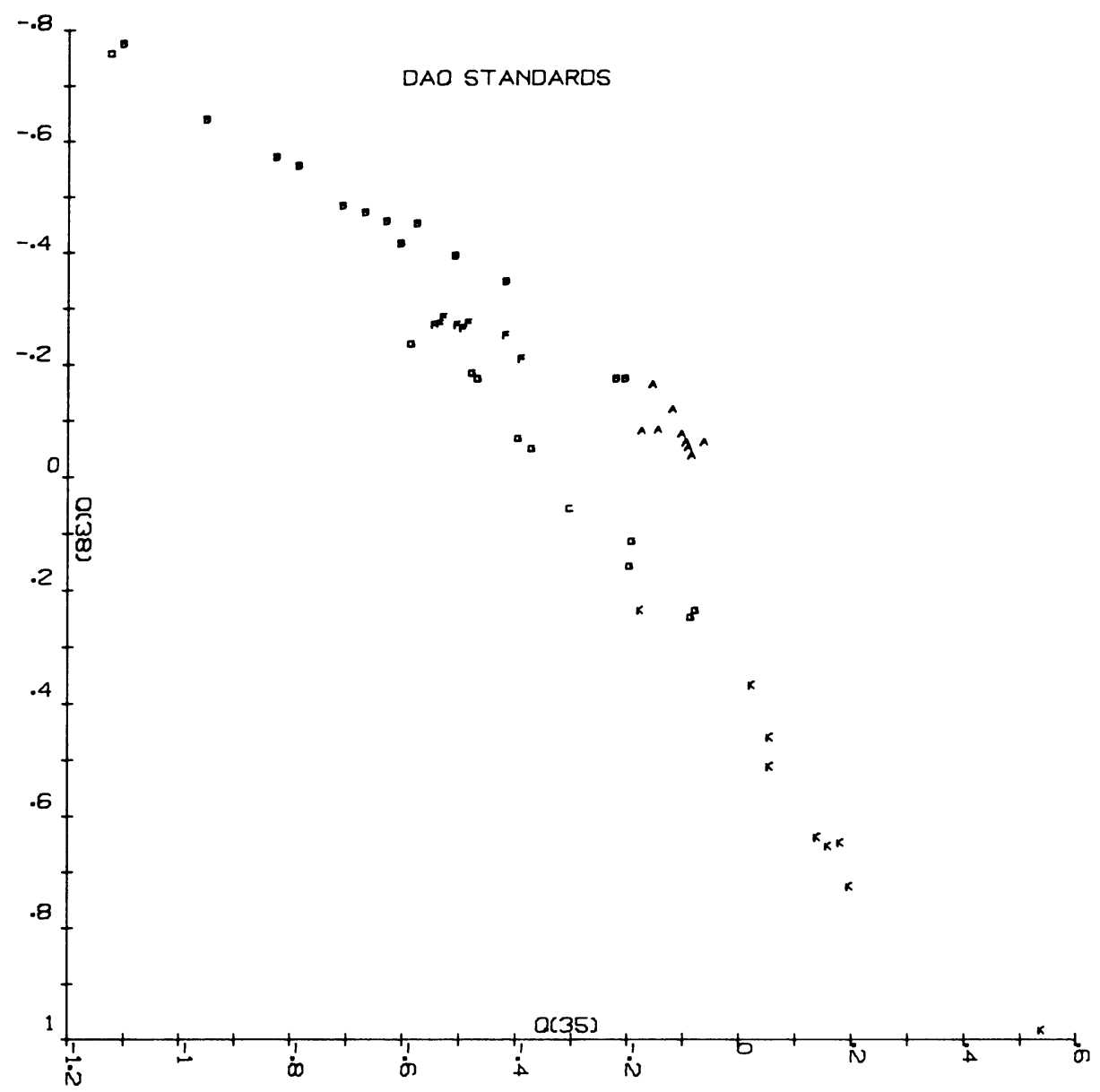

Fig. 2. $Q-Q$ plot for DAO standards. The symbol plotted indicates spectral type; a composite spectrum is denoted by $c$.

and

$$
Q(38)=c(38-44)-\frac{E(38-44)}{E(44-54)} \cdot c(44-54)
$$

We assume that the ratios of the colour-excesses are constant around the sky. In Figure 2 we show a ' $Q-Q$ ' plot $[Q(35)$ vs $Q(38)]$ for the DAO standards, for stars ranging in spectral type from late $\mathrm{O}$ to late $\mathrm{K}$. It is clear that this classification method 


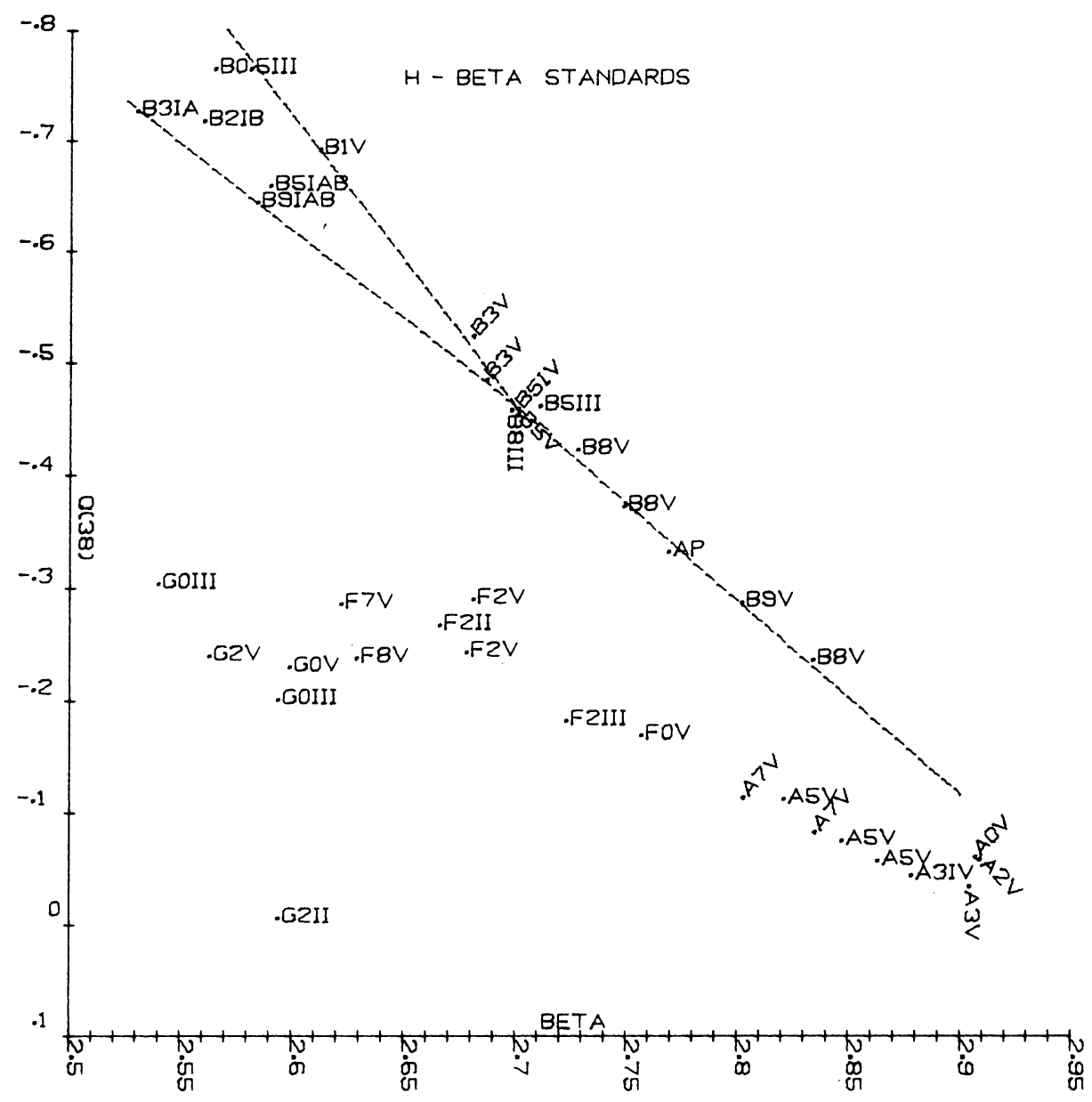

Fig. 3. The relation between $Q(38)$ and $\mathrm{H} \beta$ index.

will probably not be suitable for types $\mathrm{A}$ and $\mathrm{F}$. The relation between what we expect to be the luminosity sensitive index $Q(38)$ and Crawford's $\beta$-index (Crawford and Mander, 1966) is seen in Figure 3. For luminosity class V stars there is a linear relation from B0-B5 and another linear relation from B5-B8. The change of slope is probably caused by the inclusion of a number of $\mathrm{He}_{\mathrm{I}}$ lines, principally $\lambda 3819$, in the 38 band. This line tends to strengthen as the $\mathrm{H}$ lines weaken in the earlier main-sequence stars. The supergiants follow a different line. In these stars, $\lambda 3819$ varies much less 


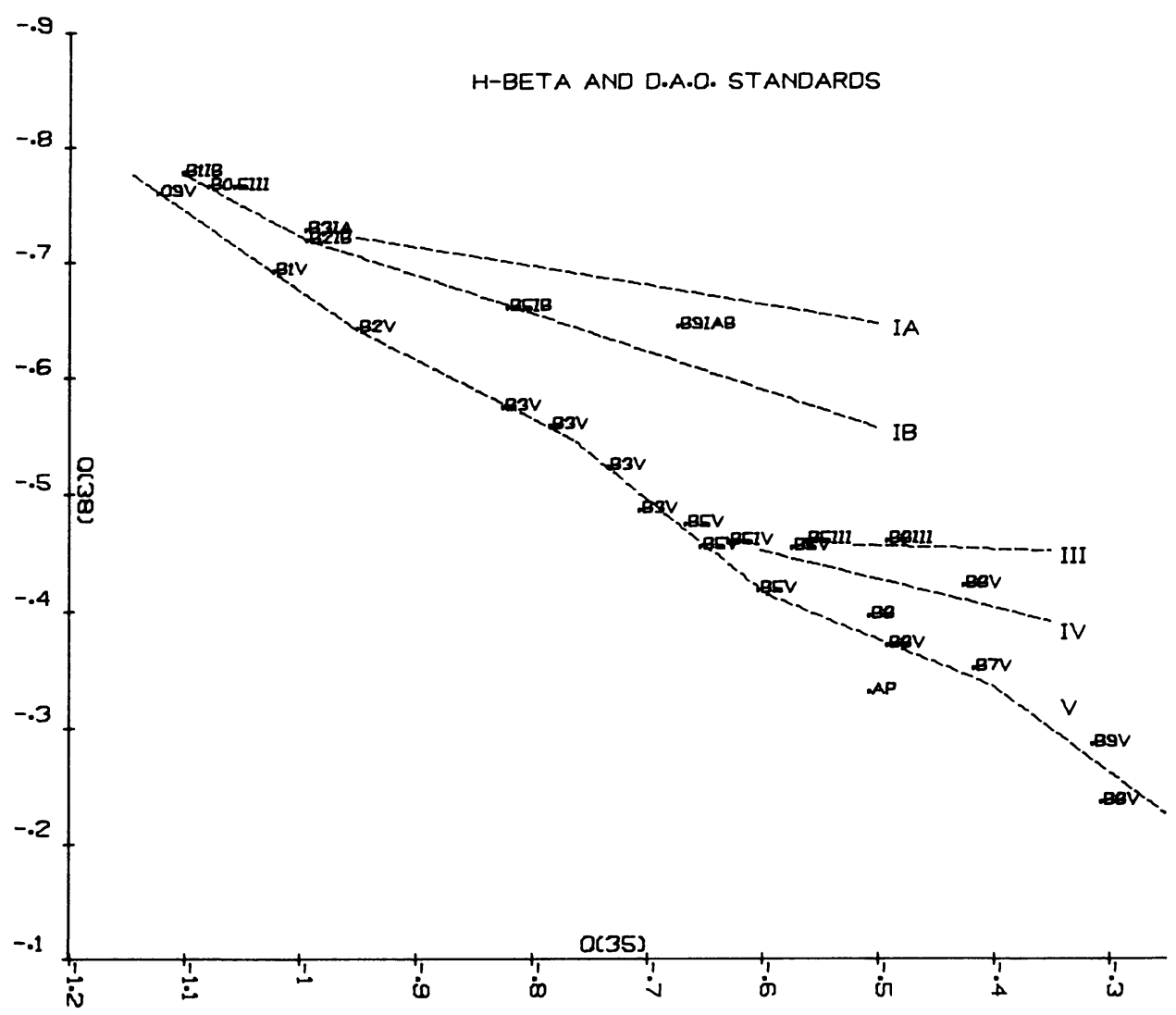

Fig. 4. $Q-Q$ plot showing spectral types and luminosity classes.

rapidly with temperature and disappears from the spectrum somewhere between $\mathrm{O} 5$ and 08.5. If one plots measures of Be stars on this diagram,'most of them fall on a line parallel to, but about 0.2 below, the extension of the class $\mathrm{V}$ line. Figure 4 is a $Q-Q$ plot for the D.A.O. standards and our observations of Crawford's $\mathrm{H} \beta$ standards, both of which groups have been exceptionally accurately observed. The main sequence line is very well defined although a little irregular; the loci of the other classes is indicated, but is based on as yet inadequate data. Nevertheless, it seems clear that a 


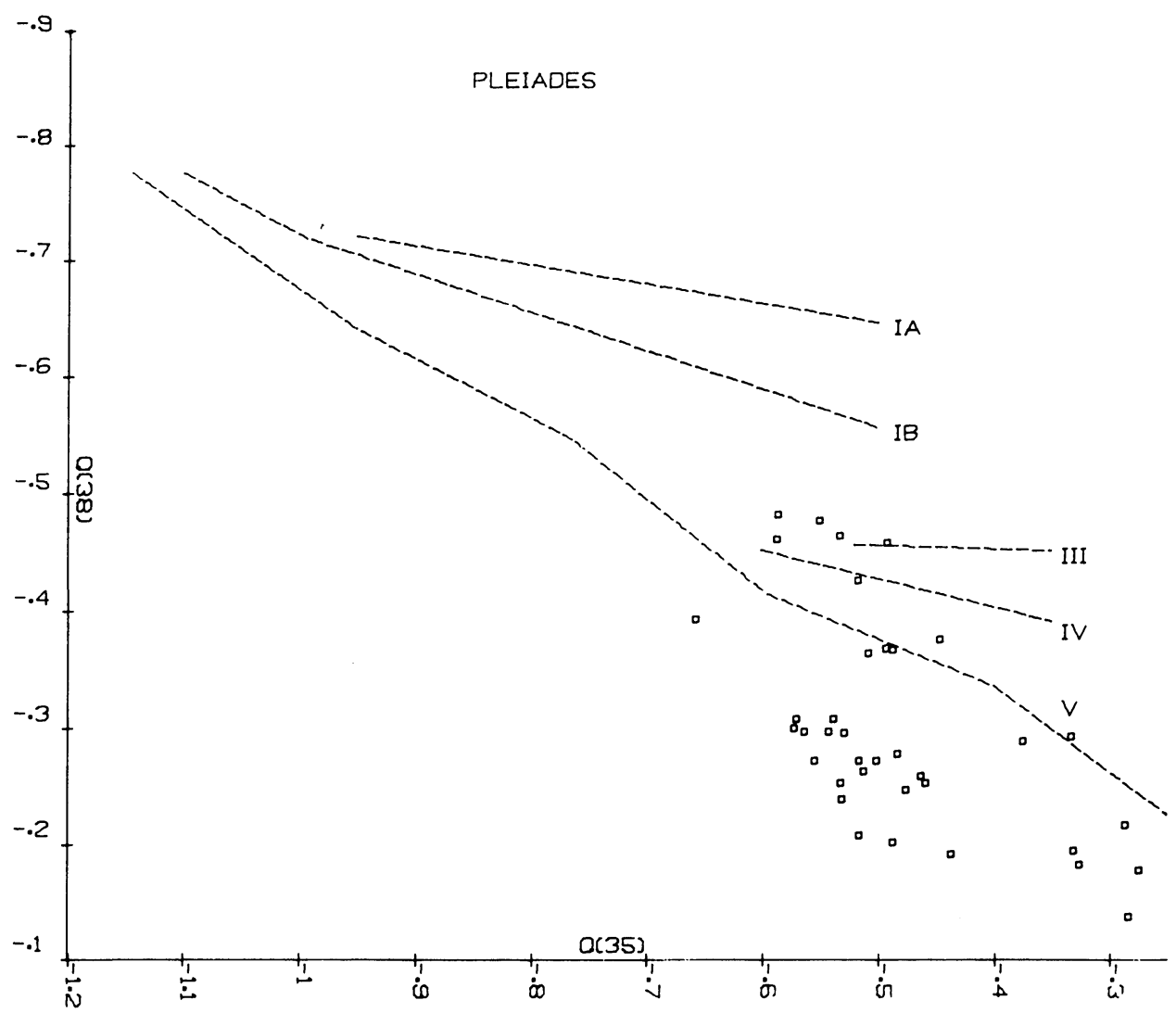

Fig. 5. $Q-Q$ plot for the Pleiades. In this and succeeding figures the luminosity class lines are those derived in Figure 4.

good calibration will be possible with the additional data that we have in hand. The wide separation of the luminosity classes in the $Q-Q$ plane is noteworthy and indicates that this method should yield good classification accuracy.

We now compare observations of various clusters with the preliminary calibration. The cluster observations are displayed on a background derived from Figure 4. Figgures 5-7 show results for the Pleiades, NGC 7243 and NGC 2169. The similarity found here between the Pleiades and NGC 7243 agrees with the results of Hill and 


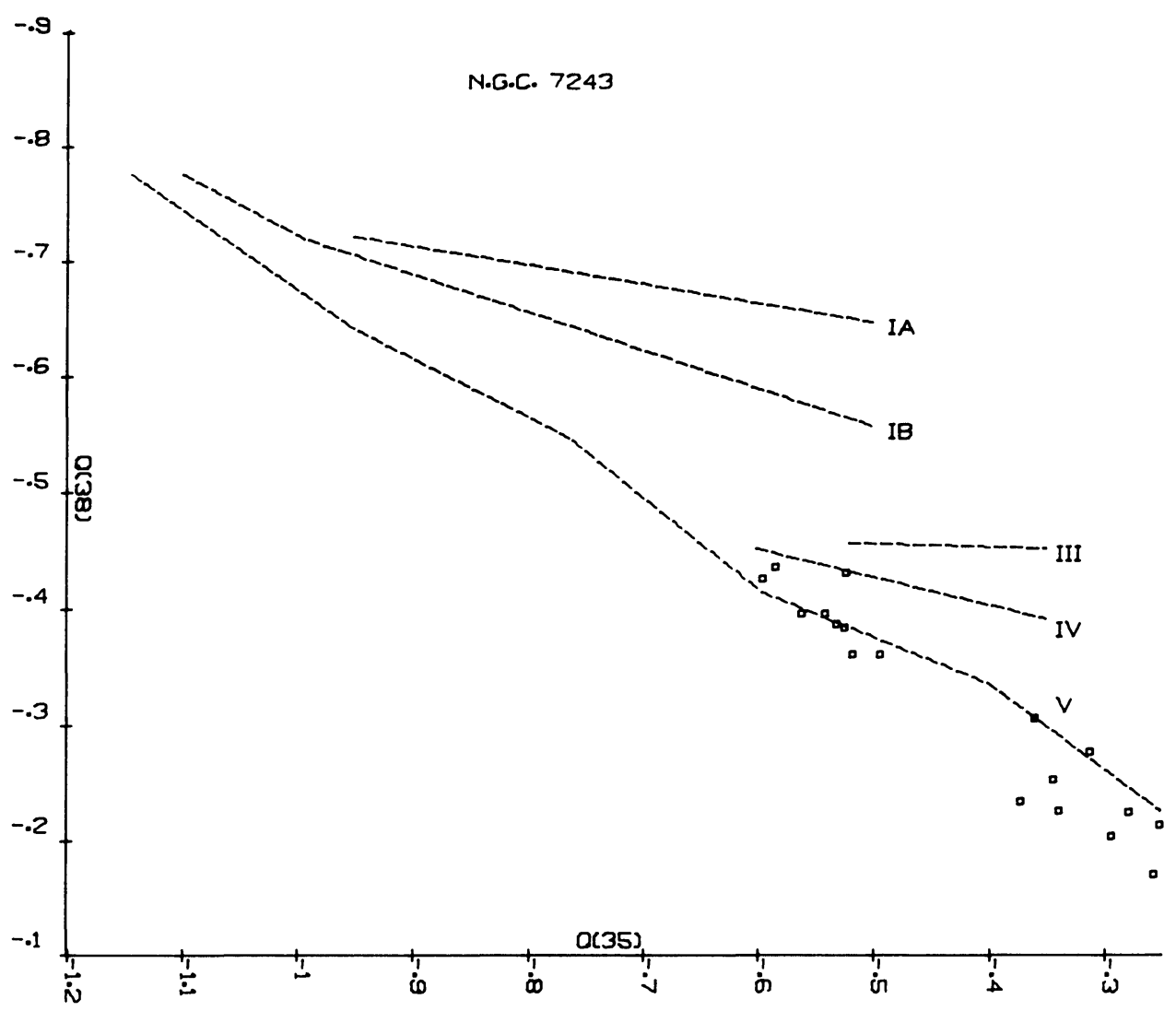

Fig. 6. $Q-Q$ plot for NGC 7243 .

Barnes (1971); in particular, very similar ages are indicated by the positions of the turnoff points. NGC 2169 is seen to be a young, relatively un-evolved cluster. In $h$ and $\chi$ Persei (Figure 8) the transition from class $\mathrm{V}$ to class $\mathrm{Ia}-\mathrm{Ib}$ is notable. A number of deviant points is apparent. Those for which we have spectral types are emission-line objects, or, in one case, an A1Ia star. Cepheus IV is interesting (Figure 9) because of an undoubted systematic deviation from the class V line. Such a difference could be caused either by anomalous values for the ratios of the colour excesses in the direction 


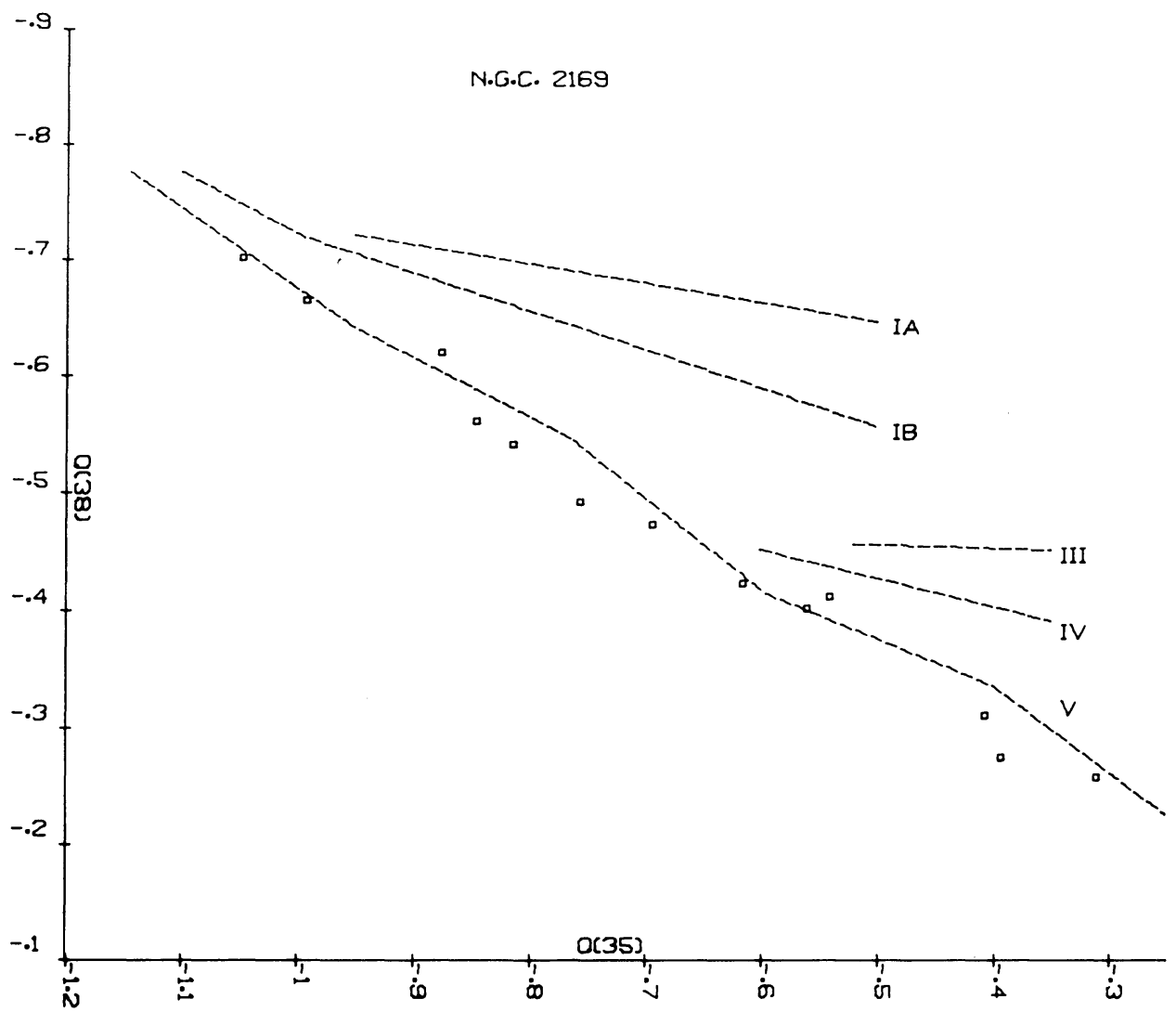

Fig. 7. $Q-Q$ plot for NGC 2169 .

of Cepheus IV, or by the stars in the association really being fainter than class V. Measures of these stars plotted in Figure 1 appear to define an extension of the normal reddening line (as is also the case for the $c(38-44)$ vs $c(44-54)$ plot); this is evidence against anomalous reddening in the direction of the cluster. Walker (1965) examined the luminosity sensitive $\mathrm{He}$ II line, $\lambda 4686$, in the spectra of members of Cepheus IV and found evidence that the stars are subdwarfs, thus supporting the second possible explanation. From its involvement with extensive nebulosity, this 


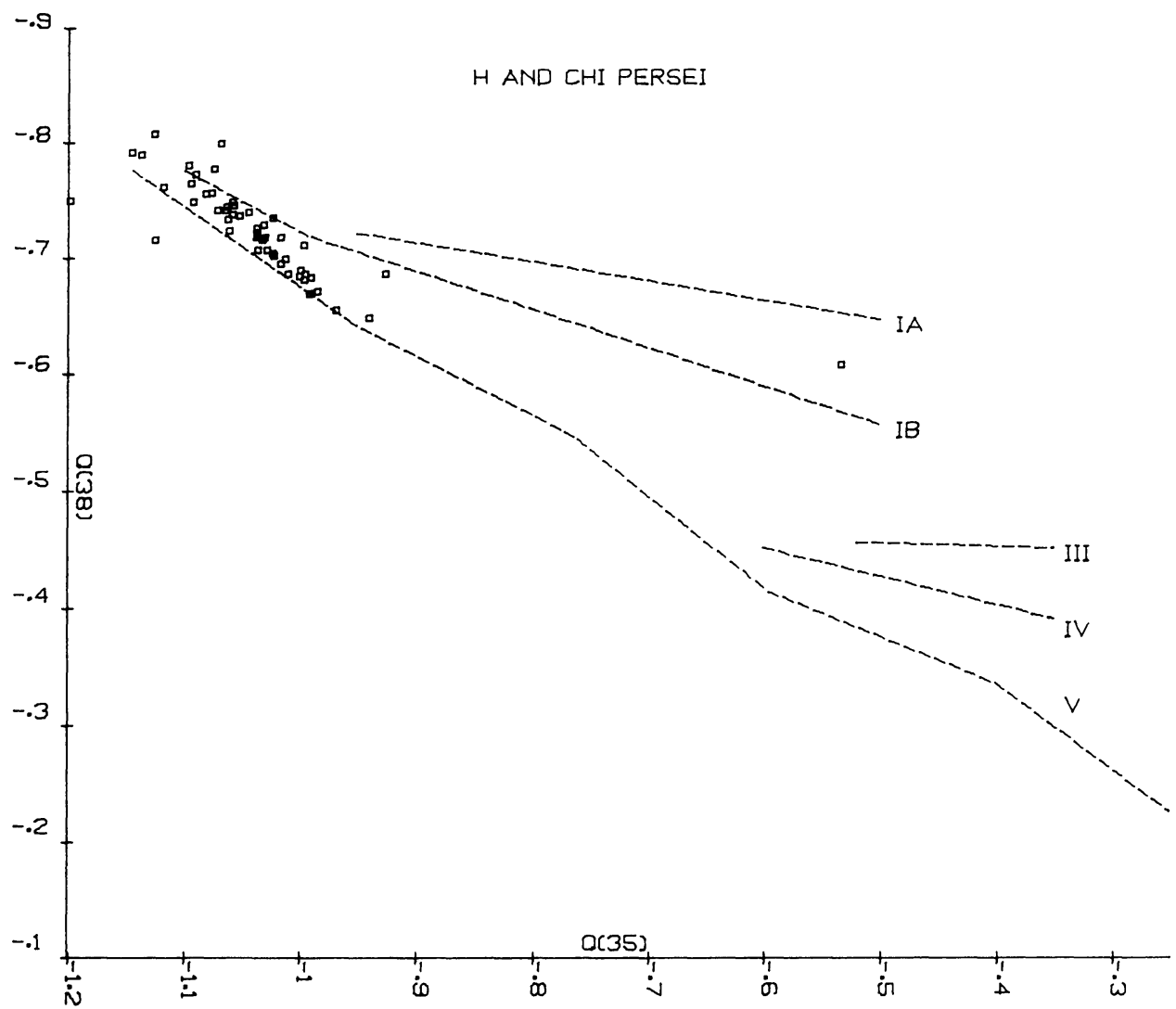

Fig. 8. $Q-Q$ plot for $h$ and $\chi$ Persei.

group of stars appears to be very young. During its hydrogen-burning lifetime, a B star will brighten by about one magnitude. Thus the class $\mathrm{V}$ line in the plots should have a breadth of about one magnitude, and the irregularity of the class $\mathrm{V}$ line in our plot for standard stars is a result of defining it by stars with a variety of ages. The deviation of measures of stars in Cepheus IV from the line defined by measures of standards is thus probably an age effect. 




Fig. 9. $Q-Q$ plot for Cepheus IV.

\section{References}

Crawford, D. L. and Mander, J.: 1966, Astron. J. 71, 114.

Hill, G. and Barnes, J. V.: 1971, Astron. J. 76, 110.

Hill, G., Morris, S. C., and Walker, G. A. H.: 1971, Astron. J. 76, 246.

Johnson, H. L., Mitchell, R. I., Iriarte, B., and Wisniewski, W. Z.: 1966, Commun. Lunar Planetary Lab. 4, 99.

Walker, G. A. H.: 1965, Astrophys. J. 141, 660.

Walker, G. A. H., Andrews, D. H., Hill, G., Morris, S. C., Smyth, W. G., and White, J. R.: 1970, Publ. Dominion Astrophys. Obs. 13, 415. 